Activités

3-2 | octobre 2006

Varia

\title{
La référence au travail en classe de technologie : un débat de métier
}

The reference to work in technological classroom: a professional debate

Régis Ouvrier-Bonnaz et Christiane Werthe

\section{(2) OpenEdition}

\section{Journals}

Édition électronique

URL : http://journals.openedition.org/activites/1325

DOI : $10.4000 /$ activites. 1325

ISSN : 1765-2723

Éditeur

ARPACT - Association Recherches et Pratiques sur les ACTivités

Référence électronique

Régis Ouvrier-Bonnaz et Christiane Werthe, «La référence au travail en classe de technologie: un débat de métier», Activités [En ligne], 3-2 I octobre 2006, mis en ligne le 15 octobre 2006, consulté le 02 mai 2019. URL : http://journals.openedition.org/activites/1325; DOI : 10.4000/activites.1325

\section{(c) (i) (9)}

Activités est mis à disposition selon les termes de la licence Creative Commons Attribution - Pas d'Utilisation Commerciale - Pas de Modification 4.0 International. 


\title{
La référence au travail en classe de technologie : un débat de métier
}

\author{
Régis Ouvrier-Bonnaz \\ Équipe de psychologie de l'orientation, INETOP, \\ CNAM, 41 rue Gay Lussac, 75005 Paris \\ ouvrier-bonnaz@cnam.fr
}

Christiane Werthe

Équipe clinique de l'activité, Laboratoire de psychologie du travail et de l'action,

CNAM, 41 rue Gay Lussac, 75005 Paris

werthe@cnam.fr

\begin{abstract}
The reference to work in technological classroom: a professional debate. Distinguishing between two concepts : the working community's "collective "trade memory" and one's own private "personal memory of work". This paper presents an analysis of a dialogue between two technology education teachers during a cross-self-confrontation session. It strives to show that in dialogue among peers sharing the same profession and researchers, resorting to one's trade memory and professional genre favours the development of interpretations concerning professional rules, thus reviving and preserving the discussion of professional issues thought to be no longer relevant.
\end{abstract}

\section{KEYWORDS}

Activity, dialogue, experience, genre, profession, work.

Dans cet article, l'activité de professeurs de technologie au collège est prise comme exemple pour aborder la question du travail enseignant à partir d'un point précis des programmes de la discipline : l'utilisation de références prises dans le monde du travail pour installer des activités scolaires. La notion de pratique de référence a été proposée dès 1981 par Martinand dans le cadre de la redéfinition des programmes de technologie au collège. Il s'agissait de prendre en compte pour organiser la discipline non seulement les savoirs en jeu mais aussi les objets, les instruments, les problèmes et les tâches, les contextes et les rôles sociaux et d'analyser les écarts entre les activités scolaires susceptibles d'être proposées et les pratiques socio-techniques prises comme référence (Martinand, 1986, p.137-138). ${ }^{1}$ Dans une recherche portant sur les pratiques enseignantes en technologie au collège (Lebeaume, 1999), les chercheurs concluent que peu d'enseignants interrogés, alors qu'ils considèrent comme

1 L'étude présentée dans cet article s'inscrit dans le cadre d'une recherche de l'Institut National de Recherche Pédagogique sur la connaissance du travail au collège. Recherche 30772 : Analyse du travail et connaissance du monde professionnel au collège : transformer et coordonner les approches en technologie et en orientation (2001/2004) en partenariat avec le Bureau des Innovations du ministère de l'Education Nationale et l'INETOP (CNAM). Neuf enseignants de technologie de collège issus de trois académies ont participé à cette recherche pendant trois ans. L'analyse du travail présentée ici, a été conduite avec deux enseignants de la même académie, au sein d'un petit collectif de quatre personnes engagées dans la recherche. 
leur devoir « d'appliquer les programmes », évoquent la notion de référence pourtant centrale dans l'organisation de l'enseignement de la technologie. Ils précisent que l'intégration « des références socio-techniques » est particulièrement délicate. Si ce constat est déterminant pour la compréhension de ce qui fait la réalité de l'enseignement de la technologie au collège, l'enquête nous apprend peu de choses sur l'analyse des difficultés rencontrées par les enseignants pour intégrer la question de la référence dans leur pratique et en débattre comme en témoigne le propos d'un enseignant : « dans la production d'un service, il ne me semble pas qu'il y a matière à nourrir le travail d'une classe de 21 élèves, je n'y arrive pas ; si je voulais que les activités soient représentatives de ce qui se passe dans la réalité, j'avais pas besoin de tout ce monde parce qu'en fait je trouve que l'effectif est nombreux ... on ne demande pas à chaque secrétaire de faire la même lettre. C'est ça le problème ... » (Lebeaume, 1999, p.76). L'enseignant est ainsi mis en situation de verbaliser les contraintes vécues dans son activité. Le tête-à-tête avec le réel mobilisé dans l'entretien d'enquête, s'il a bien permis d'envisager l'organisation du travail de l'enseignant en technologie comme la source de son activité, permet plus difficilement de l'envisager comme une ressource laissant voir ce qui se fait réellement pour en faire un réel objet de débat entre les professionnels. L'absence de travail d'analyse à partir de traces de ce qui est fait en situation et ce qu'en disent les intéressés conduit le plus souvent les chercheurs, faute de données précises sur le détail de l'action, à réduire la description de l'activité enseignante à des listes de difficultés et des recueils de points forts ou faibles. À quelles conditions, l'expérience peutelle être considérée comme une ressource qui permette aux enseignants de réfléchir à ce qu'ils ont fait ou auraient pu faire en situation pour répondre à leurs obligations de travail ?

Les méthodologies développées dans le champ de la psychologie du travail se servent traditionnellement de l'analyse de l'activité comme d'un instrument de connaissance des situations de travail pour améliorer les conditions d'exercice du métier. L'analyse de l'activité a alors un statut d'instrument de connaissance tournée vers l'activité du chercheur. Il s'agit de comprendre le travail pour le transformer. L'analyse de l'activité peut aussi changer de statut et devenir un instrument de connaissance du travail de professionnels. Elle peut ainsi devenir un instrument psychologique au sens donné à la notion d'instrument par Vygotski (1930/1985) mis à la disposition des travailleurs pour comprendre ce qu'ils font. Dans cette logique, le travail d'analyse ne repose plus sur l'interprétation de la situation par le chercheur considéré comme expert mais sur le développement de l'interprétation de la situation chez les enseignants eux-mêmes. Comme nous essayerons de le montrer, l'interprétation de la situation par les enseignants eux-mêmes permet de relancer des débats de métier, considérés comme épuisés entre eux.

Le dispositif méthodologique qui sert de support à notre analyse a été mis en place dans le cadre des travaux de l'équipe clinique de l'activité du laboratoire de psychologie du travail et de l'action du CNAM (Clot, Faïta, Fernandez, \& Scheller, 2001 ; Clot, Prot, \& Werthe, 2001). Dans le domaine éducatif, il a donné lieu à un développement auprès d'acteurs de la validation des acquis dans l'éducation nationale (Clot, Magnier \& Werthe, 2000), et d'enseignants (Clot et al., 2005). Nous en rappelons quelques principes.

\section{1.- Clinique de l'activité : quelques éléments de méthodologie}

La méthode d'autoconfrontation croisée repose sur une conception précise de l'activité de travail : l'activité est dirigée vers son objet et simultanément vers l'activité d'autrui, elle-même tournée vers cet objet. La méthodologie mobilisée en clinique de l'activité s'appuie sur l'idée que l'activité réalisée n'est jamais que l'actualisation d'une des activités réalisables dans la situation où elle voit le jour. Dans cette situation, le développement de l'activité qui a vaincu (Vygotski, 1994) est gouverné par les conflits entre les activités concurrentes qui auraient pu réaliser la même tâche à d'autres coûts. Ainsi, « le réel de l'activité c'est aussi ce qui ne se fait pas, ce que l'on cherche à faire sans y parvenir - le drame des échecs - ce que l'on aurait voulu ou pu faire, ce que l'on pense pouvoir faire ailleurs. Il faut y ajouter - paradoxe fréquent - ce que l'on fait pour ne pas faire ce qui est à faire» (Clot, 1999, 
p. 119). L'expérience professionnelle n'est pas un produit fini d'où l'idée avancée en clinique de l'activité que l'expérience ne se limite pas à ce qu'on sait faire mais englobe également les possibilités dont le professionnel dispose ou pas pour se défaire des situations auquel il se confronte (Clot, 2004). L'activité retirée, occultée ou repliée, n'est pas absente, elle pèse de tout son poids dans l'activité présente. Dans cette logique, «l'expérience vécue n'étant pas directement accessible par l'analyse, c'est à l'aide des traces qu'il faut construire ou plutôt co-construire avec nos interlocuteurs. Ces traces, objet controversé d'un dialogue, peuvent alors devenir les sources d'une élaboration conjointe » (Diallo, \& Clot, 2003, p. 206).

Plus qu'une méthode, il s'agit d'installer une méthodologie de co-analyse qui associe les protagonistes d'une situation de travail à son analyse, afin de les seconder dans leurs efforts pour comprendre leur manière de faire et élargir leur pouvoir d'action. Il s'agit alors de favoriser la confrontation de professionnels avec leur métier pour qu'ils puissent se reconnaître dans ce qu'ils font en s'inscrivant dans une histoire professionnelle. Ce qui est recherché, c'est le rappel d'une mémoire collective constitutive du métier, sorte de répondant collectif de l'activité individuelle. Dans l'autoconfrontation, l'objectif est de faire de cette mémoire collective fondatrice de ce qui est nommé en clinique de l'activité, le genre professionnel, une ressource pour penser l'action en milieu professionnel. Quels liens peut-on alors établir dans le domaine de l'analyse du travail entre mémoire collective du métier et mémoire privée du travail ? Les enjeux que soulève cette question mérite qu'on s'y attarde un peu. Nous le faisons en discutant la méthode d'entretien d'explicitation proposée par Vermersch et le concept de pensée privée qu'il distingue de la pensée socialisée qui suppose une mise en mot pour l'autre (1993, p. 211).

\section{2.- Explicitation de l'expérience et mémoire privée du travail}

L'apport de Vermersch est une contribution importante à l'analyse de l'action par la verbalisation. L'entretien d'explicitation se présente comme une technique qui permet la mise à jour du fonctionnement cognitif des sujets engagés dans l'action à partir de leur propre langage. Cette technique consiste à permettre au sujet d'évoquer le comment de son fonctionnement dans une situation de référence par un guidage précis de ses verbalisations. Le sujet doit faire un effort de mémoire pour se remémorer la situation et évoquer le déroulement des opérations mentales mobilisées au cours de la réalisation de la tâche. Le souci de la recherche de précision, la proximité avec les détails de l'action caractérisent cette démarche. Le rappel des faits en mémoire conduit à retrouver les indices gestuels, kinesthésiques qui permettent de reconstruire le déroulement de l'action sur le plan comportemental et la succession des opérations mentales. Au départ, ce qui est fondamental, c'est de définir une catégorie de non-conscient qui est conscientisable (Vermersch, 1994, p. 77). Avant d'être connu, le vécu qui sera l'objet de la verbalisation doit être reconnu par le sujet (Vermersch, 2005, p. 47). Ce principe de « reconnaissance de soi à soi » est la condition de l'activité de réfléchissement à la base de l'explicitation phénoménologique qui structure l'approche de Vermersch. Dans ce cadre, la pensée privée à laquelle seul le sujet a un accès direct recouvre deux aspects : celui des actions mentales et la manière dont le sujet se représente un contenu donné lié à une action finalisée (Vermersch, 1993, p. 211). La source d'information est alors ce que le sujet peut dire de son expérience, ce qui est conscientisable de son expérience (p. 212).

Dans l'entretien d'explicitation, Vermersch reprend l'idée qui introduit l'œuvre de Piaget : l'action est un savoir autonome et il existe un décalage entre ce qui relève de la réussite et ce qui relève de la compréhension. Entre réussir et comprendre, il y a un décalage qui fonde une théorie de la prise de conscience. De cet emprunt, découle la reprise du concept de réfléchissement et les méthodes d'analyse clinique introspective propres à la démarche piagétienne. L'évocation de l'expérience vécue dans l'explicitation ne serait pas sa simple réitération mais pourrait être vue comme la transformation de l'expérience. Vermersch indique alors qu'il serait plus juste de parler de « création de mémoire » (1994, p. 102) qui est « une mise en mot pour l'autre » à partir d'un vécu préalablement reconnu et 
restitué par rapport à la motivation qui l'initie.

Le réfléchissement est au point de départ de l'opération cognitive qui conduit à la prise de conscience, il permet la représentation de ce qui a été fait. C'est cette représentation et plus précisément encore le produit de l'action de se représenter ce sur quoi porte l'action finalisée que la pensée verbale pourra prendre ensuite comme objet. Pour Vermersch, il est nécessaire de distinguer l'activité réfléchie de l'activité réfléchissante. «La première porte sur des données déjà conscientisées, elle est une réflexion - sur -, elle est largement synonyme du sens banal de réfléchir : prendre pour objet de pensée d'autres objets de pensée(donc déjà disponibles). La seconde au contraire est au sens de Piaget (1977) - réfléchissement - c'est-à-dire passage du vécu en actes au plan de la représentation de ce vécu, préalable à la possibilité de l'exprimer, elle est réflexion de » (Vermersch, 1999, p. 14). En référence aux travaux de Léontiev (1984) sur la liaison entre l'activité et la conscience, Vermersch (1994) précise que le réfléchissement dans l'évocation n'est pas assimilable à un simple reflet mais doit être considéré comme un produit, projection d'une réalité d'un plan sur un autre plan, pensée sans cesse reprise et déplacée du vécu à l'utilisation des mots qui le caractérise. C'est dans ce mouvement que résiderait la possibilité pour le sujet de décrire l'action et de la comprendre. La verbalisation est subordonnée à la prise de conscience. Vermersch (1999), en séparant l'activité langagière du réfléchissement, laisse de côté l'idée que la verbalisation est un instrument de la prise de conscience et qu'elle ne peut donc pas précéder celle-ci. Comme le signale Prot, discutant l'idée avancée par Vermersch de « suspension » des gestes mentaux pour les prendre comme objets de questionnement et en faire des contenus d'évocation, «le réfléchissement devient un accueil de l'expérience passée. La verbalisation vient ensuite » (2003, p. 59). Dès lors, «l'acte d'évocation finit par être étudié en lui-même, la conscience devient une activité autonome, conçue en dehors des buts et des intentions que le sujet réalise au moment même où il constitue son expérience comme objet de pensée » (p. 60).

\section{3.- Mobiles de l'action et mémoire collective du métier}

L'approche de Vermersch laisse alors délibérément de côté, en se focalisant sur le déroulement procédural et opératoire de l'action, le problème de sa formation et de son intentionnalité, c'est-à-dire des buts qu'elle poursuit et des mobiles qui la sous-tendent dans un contexte de développement. L'action ne se limite pas à la seule réalisation de la tâche et à l'évocation des opérations vécues comme rappel d'événements passés indépendants des intentions présentes du sujet à l'égard des autres et de luimême, même à son insu. S'il s'agit bien comme l'indique Vermersch d'une « création de mémoire », processus de passage du vécu au représenté, il faut l'envisager comme une « création psychologique nouvelle » (Clot, 2000) qui ne soit pas seulement un représenté d'un vécu antérieur mais aussi un représentant du vécu présent du sujet dans son rapport avec son ou ses interlocuteurs : le produit psychologique d'une situation d'interlocution socialement et historiquement définie. Une nouvelle représentation est ainsi construite grâce à la médiation de la situation interlocutoire vécue, le destinataire de la verbalisation agissant sur les contenus. Ce n'est donc pas dans la vie propre de chacun que le vécu prend sa signification mais dans la confrontation avec des « points de fixation de sens » situé hors du contexte de la vie propre (Bakhtine, 1984, p. 123). Pour reprendre cette perspective théorique dans nos propres termes, ce n'est pas dans la mémoire privée du travail que le vécu professionnel prend sa propre signification mais dans la mémoire collective du métier.

Pour Bakhtine, « comprendre... c'est penser dans un contexte nouveau » (1984, p. 384) l'approfondissement du sens se faisant par " élargissement des distances contextuelles ». Ce que dit Vygotski d'une autre façon : «l'action passée au crible de la pensée se transforme en une autre action, qui est réfléchie » (1994, p. 227). Expliquer au chercheur, c'est prendre un point de vue nouveau sur son travail. Prendre conscience ne consiste donc pas seulement à retrouver par et en pensée un passé intact de toute modification mais à le faire revivre dans et par l'action présente en fonction d'objectifs qui ne dépendent pas seulement de la tâche évoquée mais de ce qui incite le professionnel à agir au moment de l'analyse en regard de l'histoire du métier : celle que chaque professionnel va parvenir 
ou non à faire sienne en y mettant du «sien ». Du coup, si on adopte cette posture méthodologique, les motifs ne peuvent être séparés de l'explicitation de ce qui a été fait et l'évocation du vécu ne peut être considérée seulement comme une affaire privée. Ce point de vue méthodologique est à la base de la méthode d'autoconfrontation croisée utilisée en clinique de l'activité.

\section{4.- Dialogue entre les deux professeurs : la référence au travail en classe de technologie en collège}

La partie du dialogue, présentée ci-dessous, a été choisie en accord avec les deux enseignants concernés pour deux raisons : elle porte sur la question de la référence considérée comme élément central de l'organisation des programmes de technologie au collège et des activités proposées aux élèves en classe et elle concerne un point souvent débattu entre les deux enseignants qu'ils pensaient avoir épuisé. Ces deux enseignants, PL et CD, peuvent être considérés comme des experts de même niveau de l'enseignement de leur discipline. Depuis plusieurs années, ils conduisent des activités de formation en direction d'autres professeurs de technologie et ont participé à des groupes de réflexion sur la mise en place des programme de la discipline. L'extrait de dialogue pris dans sa continuité peut donner une idée des effets attendus concernant le déplacement des points de vues dans le dialogue.

Les deux enseignants (PL et CD) ont été filmés pendant une heure chacun dans une classe de quatrième de leur collège.

La séquence que nous nous proposons d'examiner porte sur 3 minutes d'activité conduite par un professeur PL dans une salle multimédia. Il s'agit d'un cours en classe entière. L'enseignant a monté dans le collège une "mini entreprise» de sous-traitance en liaison avec une entreprise de recyclage de cartouches d'encre pour imprimante de la région. Cette activité s'inscrit dans un scénario, production de service « qui court» tout au long de l'enseignement. Un scénario dans les programmes de technologie du cycle central du collège (classe de quatrième et de troisième) est considéré comme « une mise en scène » dans la classe d'un ensemble de références choisies dans le monde professionnel pour réaliser et produire à l'école. Dans la démarche mise en œuvre par l'enseignant, les élèves sont amenés à découvrir certains documents propres au fonctionnement d'une entreprise (bordereaux, factures, ...) et les fonctions professionnelles qui leur sont attachées. Dans la séquence commentée ci-dessous, l'enseignant, après les consignes de début de classe (appel, mise en route des ordinateurs, ...), présente une enveloppe en provenance de l'entreprise de recyclage reçue le matin même au collège et que la concierge lui a remis. Il la tend à un élève, lui demande de l'ouvrir et d'identifier sa provenance et sa nature à partir des éléments qui figurent sur le document placé dans l'enveloppe (un bordereau de reprise de produits usagés).

Le professeur présente à la classe l'enveloppe en la tenant « à bout de bras ».

PL : Qu'est ce que c'est? (il tend la lettre à un élève proche de lui)

(une élève intervient pour évoquer l'envoi d'un carton, cette intervention est en partie inaudible)

PL : C'est peut-être ça qui arrive... je n'ai pas ouvert l'enveloppe

C'est quoi ...(l'élève ouvre l'enveloppe)

Elève : C'est un bordereau

PL : C'est un bordereau ... ah ! (reprend la lettre et se déplace dans la classe)

C'est le bordereau de reprise... hum comme on en a déjà collé un certain nombre dans les cahiers

l'activité qu'on peut mener, c'est comparer le bordereau d'entreprise avec le bordereau que vous avez fait vous. Donc à ce moment là qu'est ce qui l'a écrit le bordereau d'envoi ? (se retourne vers les trois élèves qui ont réalisé le bordereau lors d'un cours précédent)

Il y a une qui reprend le bordereau... et qui compare... et c'est signé qui ? (montre le bordereau en le tenant devant lui à bout de bras à l'élève qui s'est levé et se dirige vers lui, place un doigt de sa main gauche à hauteur de la signature)

Elève : A... K... (nom de la personne qui a signé)

PL : Alors AK (PL se penche sur un classeur devant lui et cherche un document qu'il ne trouve 
pas).

L'activité de PL est regardée sur l'écran du téléviseur placé devant les deux enseignants. Les deux chercheurs sont placés à gauche. Le défilement de la bande peut être arrêté à tout moment à l'aide d'une télécommande par les enseignants ou les chercheurs.

L'échange entre les deux enseignants se structure autour des rapports entre les pratiques de référence et les pratiques de classe. Cette notion de référence renvoie à l'étude de l'authenticité des pratiques scolaires qui organisent l'enseignement de la technologie. L'analyse des programmes de technolo$\mathrm{gie}^{2}$, vus comme des textes prescriptifs qui peuvent être considérés comme relevant d'un genre de textes dits «d'incitation à l'action » (Plazaola Giger, 2004), montre que la recherche de cohérence entre des pratiques prises comme référence dans le monde professionnel et les activités proposées aux élèves en classe est centrale dans le métier de professeur de technologie au collège. Cette règle est constitutive, en grande partie, du genre qui organise ce métier.

Clot (1999) définit le genre dans le domaine du travail, comme le produit de l'histoire collective de ceux qui exercent une même activité professionnelle, c'est une sorte «d'intercalaire social» entre le métier et les personnes qui le font. Le genre est à disposition de chacun et retravaillé par tous ceux qui s'y reconnaissent. Le concept de genre de métier, catachrèse empruntée à Bakhtine (1984), est ainsi déplacé dans le champ de l'analyse du travail. Pour cet auteur, il existe des genres de discours stabilisés construits historiquement permettant de prendre place dans les échanges sociaux et de les développer. Appliqué au travail, il renvoie à ce que doit connaître et maîtriser une personne pour entrer et être reconnue dans une communauté de travail donnée.

Le genre se présente comme une ressource pour le métier qui vient donner «corps » à l'exercice professionnel en permettant au sujet de « se tenir et d'être tenu » aux et par les règles d'action et les valeurs constitutives du métier. L'enseignant de technologie n'est pas seul face aux prescriptions institutionnelles. Il appartient à un collectif dont l'histoire a produit et construit des façons de faire et de penser propres à une communauté que les questionnements, les désaccords, les tensions, les reprises des justifications premières dans le dialogue viennent réactiver.

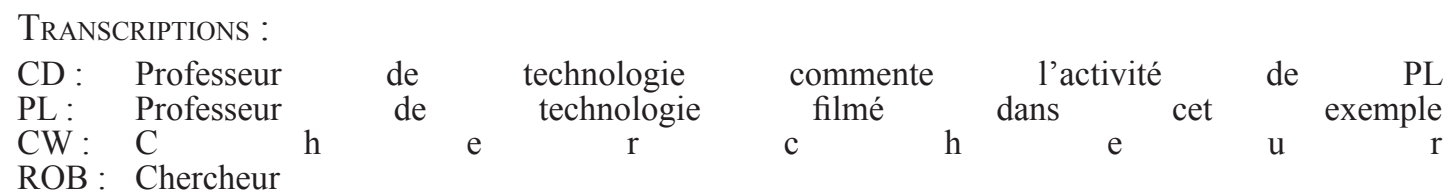

1. ROB à CD: Ta question, c'était... visait à dire pourquoi c'est pas l'élève qui a ouvert, qui lit le bordereau, qui dit c'est signé...

2. CD : Oui... oui, en pareille circonstance, je pense que j'aurais... mais, je ne sais pas, c'est pas le même public. Bon, imaginons en pareille circonstance, je pense que j'aurais fait jouer le rôle à l'élève jusqu'au bout, quitte à ce qu'il se trompe, c'est-à-dire que les autres lui disent c'est pas ça, ou je sais pas quoi euh,... je trouve l'élève trop passif pour moi. J'aurais spontanément, j'aurais rendu l'élève plus acteur. Mais c'est qu'une impression...parce qu'il dit l'élève ce que c'est. En fait, il identifie le document, etc. Tu fais venir l'élève qui est responsable du bordereau d'envoi... 3. PL : J'accepte assez volontiers ta remarque (se recule bras croisés).

4. CD : Moi, ça, c'est quelque chose que je suis sûr... tout ça... les élèves ont le sentiment de vivre et d'appréhender, mais quelque part, c'est quand même toi qui le fais, enfin, c'est peut-être quelque chose qui nous différencie... Je peux me tromper.

5. CW : Est-ce que la disposition peut y être pour quelque chose sur la raison qui fait que P1

6. PL : Il y en a qui sont de dos, en plus, ils sont sur deux rangées

7. $\mathrm{CD}:$ Ça ne se prête pas tellement à ça.

8. PL : Non, ça ne se prête pas du tout à ça par rapport à la salle de technologie, le comportement est complètement différent, d'autre part, c'est une activité qui vient se surajouter à

2 Voir Bulletin Officiel de l'Education National (BOEN) : numéro hors série de 1996 portant sur les programmes de technologie au cycle central du collège. 
celle qui était prévue.

9. CD : Exact...

10. PL : Puisque l'enveloppe, on l'a trouvée le matin même. C'est la concierge qui me l'a donnée de la main à la main, le matin même, et du coup, j'ai surajouté, normalement, c'est une activité qu'on ne mène pas dans cette salle-là.

11. CD : Oui, c'est pour ça, ça a un petit côté... j'imagine très bien ça, et d'ailleurs, ça doit se produire beaucoup plus souvent, ce qu'on vient de voir là en salle, en espace de technologie, où ça a sa raison d'être. Là, c'est un peu...on rentre dans une salle multimédia, donc, pour faire de l'informatique et on ouvre du courrier, et on distribue des cahiers, on range du courrier...y a , y a quelque chose (silence) qui est inadapté, je dirais.

12. PL : Ceci dit mon scénario continue, il court, il continue...

13. CD : Non mais je suis d'accord

14. PL : Même pendant le ludo, mon scénario continue. Si j'ai un envoi à faire ou la réception d'un chèque il faut que mon scénario continue. Si j'ai un chèque à faire, il faut que l'encaissement ait lieu.

15. CD : Tout- à- fait

16. PL : C'est ce qui me différencie un peu avec toi et avec $\mathrm{C}$... (le troisième professeur de technologie du groupe). Moi, mon scénario est en cours, il continue

17. CD : C'est un fil rouge

18. CW : Donc ça se passe aussi dans la salle multimédia, et pas seulement...

19. PL : Le scénario, il continue, s'il y a un événement à gérer pendant qu'on fait le ludo, on le gère aussi pendant le ludo ... la réception d'un chèque... la séance d'avant, par exemple...

20. CD : Là, ça oblige à...

21. PL : La séance d'avant, attends, je termine, il y avait trois filles qui avaient fait un envoi, donc un bordereau parce qu'on avait effectivement un nombre de cartouches qui justifiaient un envoi. Donc trois filles avaient été retirées du ludo pour aller faire...travailler sur le scénario.

22. CD : Ce qu'il y a au niveau de l'activité et du lieu, souvent une activité est liée à un lieu identifié, dans l'espace technologique, là ce n'est pas le cas, elle est dégagée de son contexte, et ça crée chez moi un... (silence) et peut-être chez les élèves ... je ne sais pas, je ne peux pas me prononcer à leur place, mais chez moi, ça crée, non pas une espèce de gêne, ça m'interpelle, ça m'interpelle ça, c'est pourquoi on reste beaucoup là-dessus, alors que le reste, certainement va moins m'interpeller, mais ça oui.

23. CW : Tu dis toi, tu aurais fait venir l'élève à un endroit où il aurait pu parler aux autres.

24. CD : Je pense que même pas

25. CW : De sa place?

26. CD : Non, non, je pense que cette partie d'activité aurait eu lieu en bas, avant qu'on monte, parce que la prise en main a lieu en bas, on est d'accord, hum ; moi, je l'aurais laissé dans le contexte

27. CW : Ah oui!

28. PL : Le problème, c'est que je n'avais pas de place en bas,

29. CD : C'est toujours ça

30. PL : Je peux toujours le faire dans le couloir

31. CD : Quand... quand on scénarise, c'était très bien de dire, dans l'esprit, qu'il ne va pas y avoir de distorsion de temps entre l'arrivée du courrier et l'ouverture... on ne les amène pas à gérer le temps aussi, mais en même temps, c'est pas attaché à un lieu précis, alors que dans l'entreprise, si on veut modéliser quelque part, il y a un lieu, un endroit où on fait ça : classer le courrier. C'est pourquoi, ça m'interpelle, peut-être qu'avec les mêmes contraintes, j'aurais fait la même chose, c'est difficile de dire, ce n'est pas une critique négative, d'ailleurs... je n'ai pas le sentiment de le faire comme ça.

32. ROB : Tu utilises le mot scénariser (PL : oui). Précisément qu'est-ce que tu mets derrière? Ton activité que tu conduis en classe renvoie à un scénario précis?

33. CD : Pas forcément d'ailleurs dans le sens scénario techno, mais quand on scénarise, on gère du temps, on gère de l'espace, on gère de l'activité, on gère des gens... c'est tout ça que j'appelle scénariser, un peu comme au cinéma quoi... sauf que là, on ne va pas gérer de la lumière, encore que... quelques fois, ça arrive. Mais c'est tout ça qui se joue en même temps, et quelque part, quand on est dans une salle multimédia, elle a une fonction précise, la salle multimédia, on ne 
peut pas faire autre chose que ce que tu (se tourne vers PL) va faire normalement.

34. PL : C'est difficile

35. CD : Euh et on voit que l'histoire du courrier, ils sont obligés de passer par toi, tu as un classeur pour classer tout ça (gestes des bras et mains de PL), on ne le voit pas, les autres élèves ne le voient pas, il manque quelque chose pour moi

36. PL : Les élèves connaissent le classeur

37. CD : Tout-à-fait, je ne remets pas en cause tout ça, ça s'explique par le fait que tu n'as pas pu utiliser une salle de techno. Moi, je réagis par rapport à ce que je vois, et ce que je vois, c'est ce que les élèves vivent, quelque part en même temps, je me mets en situation d'élève quand je dis ça. Y a pas incohérence mais il y a quelque chose de gênant, quoi, mais c'est une adaptation aux contraintes...j'aurais sûrement pas fait mieux, j'aurais sans doute fait pareil

38. PL : Ou différemment... (en levant les épaules)

39. CD : Ou différemment, oui, différemment

40. PL : Il faut pas non plus être consensuel à tout prix (rires des enseignants et des chercheurs)

\section{5.- Dilemme de métier et développement de l'interprétation : la relance du débat professionnel}

L'objectif que nous poursuivons vise à étudier le développement de l'interprétation chez les enseignants concernant la mobilisation en classe de la notion de pratique de référence. Nous commençons l'analyse à la fin d'un long échange entre les deux enseignants sur la manière de mettre l'élève en activité en classe de technologie. Au tour de parole de l'énoncé 3, PL manifeste un accord avec CD «j'accepte volontiers » en se croisant les bras et en se renfonçant dans sa chaise. L'échange n'est pas clos, CD reprend et nomme leur différence « c'est peut-être quelque chose qui nous différencie » offrant ainsi une possibilité de poursuite du dialogue renforcée par l'intervention du chercheur en énoncé 5. L'enchaînement des précautions successives prises par CD (je ne sais pas, c'est pas le même public, c'est une impression) qui se termine en énoncé 4 par " je peux me tromper» conduit PL à se positionner, à tenir son point de vue, à le justifier, mais aussi à aller plus loin à partir de son intervention en énoncé 8 : « la salle de technologie ne se prête pas à ça... c'est une activité qui vient se rajouter aux autres ». Le lien entre les différents énoncés est ici important, c'est en effet dans le rapport des énoncés entre eux que peut être saisi et compris ce que l'interlocution réalise. L'usage que chacun des interlocuteurs fait de la parole dans l'interlocution dépend de l'activité qu'il poursuit et de ses intentions. Ainsi l'activité de CD est une activité d'analyse de ce que son collègue a fait.

$\mathrm{Au}$ contact des traces de son activité, PL se retrouve face au dilemme vécu en classe : soit différer l'ouverture de la lettre reçue le matin même au collège soit l'exploiter, le jour même, avec les élèves. Dans le dialogue installé entre les deux professionnels, ce dilemme vécu dans l'action par nature binaire (oui/non : exploiter ou non la lettre) devient un opérateur efficace dans le dialogue en se transformant en un conflit dialogique tourné vers soi, les autres et le métier où les professionnels sont délestés des préoccupations de ce qu'ils ont du ou pu faire en situation. En mettant cette activité en concurrence avec d'autres activités dans un lieu spécifique, comme le fait remarquer CD en énoncé 11 : « on entre dans une salle multimédia, donc, pour faire de l'informatique, et on ouvre du courrier, et on distribue des cahiers, on range du courrier... y a quelque chose... d'inadapté », PL prend le risque de ne pas suivre la règle. L'emploi du « on » est ici significatif, pronom indéfini, il englobe tout à la fois l'enseignant et les élèves qui ont vécu la situation et les protagonistes qui échangent sur cette situation. $\mathrm{CD}$ ne cherche pas à discuter ce que son collègue fait ou propose de faire aux élèves mais il focalise sur une activité en technologie qui va porter sur l'utilisation de la classe, définie comme un lieu de travail spécifique. L'utilisation du « on» dont on pourrait penser qu'il permet d'éviter ici l'emploi du « tu », perçu comme trop impliquant, rend discutable une question de métier sur la difficulté à gérer l'inadaptation des lieux. PL est ainsi invité à retrouver les raisons des choix qu'il a effectués en situation et des intentions qui supportent son action en reliant l'activité ponctuelle (l'ouverture d'une lettre d'une entreprise accusant réception d'un colis envoyé) proposée aux élèves 
à une activité inscrite dans la durée : la conduite d'un scénario. Il indique en 24 : «ceci dit mon scénario continue, il court, il continue ... ». Il termine en précisant en $16:$ «c'est ce qui me différencie un peu avec toi et avec C (un autre enseignant). Cette différence affirmée en appui sur convocation dans le dialogue d'un autre enseignant donne à CD la possibilité de reconnaître la complexité de la situation et le conduit à justifier l'action de son collègue concernant l'explication donnée du déroulement du scénario : «c'est un fil rouge ».

\section{1.- Le recours au métier comme ressource dans l'échange}

Dès lors, le déroulement du dialogue va permettre à PL de développer les premières interprétations, reprendre ce qui lui avait en partie échappé et que le questionnement de CD lui permet d'aborder sous un angle nouveau. Dans ce cadre dialogique, l'occasion est donnée aux deux enseignants de regarder d'un autre œil la façon dont on peut relier entre elles des activités différentes en classe de technologie dans le respect de la règle : une activité, un lieu spécifique. PL en énoncés 19 et 21 retrouve une des options possibles de l'action conduite en classe et mobilise d'autres expériences où il a proposé aux élèves, à travers la convocation du scénario, des relations avec l'entreprise de récupération de cartouches d'encre usagées. L'alternative consistait pour PL à interrompre une activité, ou plus précisément à la suspendre, la mettre entre parenthèses, le temps d'en conduire une autre. Astuce de PL pour se sortir d'une impasse? « la salle multimédia n'est pas faite pour ça » pour concilier deux activités dont l'une des deux n'aurait pas à être conduite dans le même lieu, déjà utilisée mais sous une forme différente (trois élèves avaient été soustraits de l'activité de classe pour travailler sur le bordereau d'entreprise comme PL l'indique en énoncé 21). Il convoque d'autres expériences pour lui-même et pour son collègue. Ce choix fait par PL d'ouvrir la lettre reçue de l'entreprise dans la salle multimédia, que les 2 protagonistes reconnaissent comme adapté aux contraintes, enfreint cependant une règle constitutive du métier, et ne les laisse pas tranquilles. PD insiste : « ça m'interpelle ça, c'est pourquoi on reste beaucoup là-dessus, alors que le reste, certainement va moins m'interpeller, mais ça oui ».

Le chercheur en énoncé 23 propose une alternative et demande à $C D$ ce qu'il aurait fait dans la même situation en s'appuyant sur la référence faite précédemment aux élèves. $\mathrm{CD}$, par deux fois, repousse la suggestion faite par le chercheur et conforte son point de vue: «Non, non, je pense que cette partie d'activité aurait eu lieu en bas, avant qu'on monte, parce que la prise en main a lieu en bas, on est d'accord, hum ; moi, je l'aurais laissé dans le contexte ». Il poursuit en s'appuyant sur la notion de scénario constitutive des programmes de technologie en énoncé 31 et l'utilisation qu'en fait PL : «Quand... quand on scénarise, c'était très bien de dire, dans l'esprit, qu'il ne va pas y avoir de distorsion de temps entre l'arrivée du courrier et l'ouverture... on ne les amène pas à gérer le temps aussi, mais en même temps, c'est pas attaché à un lieu précis, alors que dans l'entreprise, si on veut modéliser quelque part, il y a un lieu, un endroit où on fait ça : classer le courrier. C'est pourquoi, ça m'interpelle, peut-être qu'avec les mêmes contraintes, j'aurais fait la même chose, c'est difficile de dire, ce n'est pas une critique négative, d'ailleurs... je n'ai pas le sentiment de le faire comme ça ». Entre les tours de parole 31 et 33, on observe alors un déplacement dans le fonctionnement discursif qui passe de l'interpersonnel (les deux enseignants entre eux) au collectif des enseignants de technologie. L'activité conversationnelle de CD change de destinataire et de direction en passant de PL au collectif, réalisant alors des fonctions différentes qui vont se développer. En ne restant pas entre eux, les deux professionnels se donnent les moyens dans le dialogue de lever les sous-entendus partagés qui pouvaient leur laisser croire qu'ils en avaient fini de débattre au sujet de la notion de pratique de référence.

\section{2.- Le déroulement du dialogue et les différents registres de l'activité}

En énoncé 32, le chercheur intervient pour demander à CD de préciser la notion de scénario évoquée en $31:$ « tu utilises le mot scénariser ... précisément qu'est-ce que tu mets derrière ? : « l'activité 
que tu conduis en classe renvoie à un scénario précis ». La réponse : «quand on scénarise, on gère du temps, on gère de l'espace, on gère de l'activité, on gère des gens... c'est tout ça que j'appelle scénariser, un peu comme au cinéma quoi... sauf que là, on ne va pas gérer de la lumière, encore que... quelques fois, ça arrive. Mais c'est tout ça qui se joue en même temps », lui permet de réaffirmer sa position concernant la nécessité de s'en tenir strictement à la règle : « quand on est dans une salle multimédia, elle a une fonction précise, la salle multimédia, on ne peut pas faire autre chose que ce que tu (se tourne vers $P L$ ) vas faire normalement ». Cette dernière affirmation de $\mathrm{CD}$ suggère le déplacement de son activité de pensée du collectif à la règle, c'est-à-dire à ce qui est attendu et qui renvoie au caractère impersonnelle de la tâche. Nous faisons l'hypothèse, en appui sur les propos de Clot commentant l'apport de la théorie bakhtinienne à l'analyse des dialogues dans les autoconfrontations, que les premiers « on » convoqués en énoncé 33 dans le discours de $\mathrm{CD}$ « parle(nt) pour les manières de faire communes dans le collectif et avec elles » (Clot, 2005, p. 50). Dès lors, ce « on collectif devient objet de travail et d'interrogations » (p. 52) et s'oppose au on prescriptif impersonnel utilisé en fin d'intervention pour rappeler la règle d'utilisation de la salle multimédia. Le « on » remplit plusieurs fonctions au service de l'expression personnelle de CD dans l'interlocution : une fonction interpersonnelle qui guide l'échange avec son collègue, une fonction transpersonnelle par la convocation du métier et une dernière impersonnelle par le rappel de la règle à suivre. Dans ce mouvement de va-et-vient qui va de soi, à l'autre et au métier, chacun tente de se saisir du dernier point de vue formulé par son collègue, et réinterroge sa propre activité en classe, prenant ainsi un point de vue nouveau sur sa propre expérience. En se rapprochant et en se distinguant, l'activité de chacun des professionnels se précise et devient à nouveau discutable. L'activité dialogique développée est alors l'occasion de s'expliquer avec soi-même, les autres, le métier et les règles qui le supportent.

Dans l'échange entre les deux enseignants, l'activité interpersonnelle menace plusieurs fois de se replier sur elle-même (en énoncé 3, 22 et 31). Nous faisons l'hypothèse que les relances des chercheurs et la référence au métier " générique », marquée par l'emploi du « on » en inscrivant chacun des deux protagonistes dans la dimension transpersonnelle de l'activité leur permet de continuer à dialoguer. L'utilisation du « on » laisse entendre que, parfois, les interlocuteurs convoquent le genre comme une communauté d'évaluations propre au métier où peut être discuté ce qui apparaît comme juste et qui ne pourrait être dit autrement. S'agit-il d'un conflit de genre qui renvoie à deux façons différentes de poser la question de la référence en classe de technologie ? S'agit-il d'un écart à la règle ou d'une réélaboration de cette règle ? C'est dans la possibilité offerte aux deux enseignants de faire du métier un véritable répondant de leur action que se trouve la réponse.

\section{6.- Conclusion}

Le dialogue développé lors de cette auto-confrontation croisée a fait naître de nouvelles controverses professionnelles concernant la prise en compte de la notion de référence dans les activités installées en classe avec les élèves, à un moment où ils étaient persuadés avoir exploré toutes les facettes du métier et ne pas avoir relevé de différences majeures dans leur pratique respective. Si nous cherchons à être précis dans le travail que nous avons conduit, nous pouvons relever qu'il ne s'agissait pas de retrouver pour les identifier des procédures cognitives ou des savoir-faire modélisables et transférables. Nous avons tenté de seconder les deux enseignants pour qu'ils retrouvent les gestes techniques et les manières de faire du métier, les variations et les différences, les discordances, pour les rendre discutables, pour qu'elles deviennent des ressources au service de la compréhension de leur activité, quand ils installent en classe de technologie des activités en référence à des activités professionnelles choisies dans le monde du travail. Le développement du dialogue n'a pas été utilisé pour dégager des caractéristiques du travail enseignant perçues comme une réalité invariante. Il est utilisé pour permettre de mettre à jour comment les deux enseignants de technologie, confrontés à un dilemme professionnel non identifié comme tel au départ, redécouvrent des manières de faire et débattent de la façon dont ils réagissent face à un imprévu, ici l'inadaptation d'une salle de classe à l'exploitation 
d'un courrier en provenance d'une entreprise. Dans le dispositif installé, chacun successivement se saisit de l'explication et de l'interprétation de l'autre pour interroger sa propre conduite et l'utilisation faite de la notion de pratique de référence. Le conflit dialogique développé dans l'autoconfrontation croisée est alors utilisé comme une ressource pour développer les questionnements sur le dilemme vécu dans la classe par l'un des enseignants. «La dispute professionnelle » entre les deux enseignants permettra peut-être, en réinterrogeant l'histoire du métier, de développer l'activité personnelle en l'ouvrant vers d'autres possibles. Au-delà du constat des difficultés rencontrées dans l'exercice du métier telles qu'elles ont pu être pointées dans l'enquête citée en introduction (Lebeaume, 1999), il s'agissait dans l'échange de faire vivre le métier entre les professionnels et pour chacun d'entre eux de façon à les amener à rediscuter les questions concernant la pertinence des références à mobiliser en classe de technologie.

Nous n'avons pas cherché à réaliser une expertise du travail de ces deux enseignants ou un inventaire des difficultés et dysfonctionnements auxquels ils seraient confrontés mais à créer la possibilité de débats de métier qui leur offrent une manière nouvelle de prendre la mesure de leur expérience pour penser ce qu'ils ont à faire. Au bout du compte, la controverse engagée par les deux enseignants sur un geste professionnel en rendant à nouveau le métier discutable en fait la source possible d'une nouvelle analyse où les règles de métier sont discutées et réélaborées. Du coup, en s'expliquant avec les différences qu'ils voient à l'écran et qu'ils discutent, c'est avec le métier qu'ils s'expliquent pour en faire une ressource et en repousser les limites. Dans ce sens, analyser l'activité ce n'est pas faire en sorte qu'elle reste fixée à un contexte et à une " mémoire privée du travail », c'est au contraire la provoquer à trouver d'autres voies de réalisation en faisant appel à la «mémoire collective du métier ».

\section{RÉFÉRENCEMENT}

Ouvrier-Bonnaz, R., \& Werthe, Ch. (2006). La référence au travail en classe de technologie : un débat de métier.@ctivités, 3 (2), 15-26, http://www.activites.org/v3n2/activites-v3n2.pdf

\section{BIBLIOGRAPHIE}

Bahktine, M. (1984). Esthétique de la création verbale. Paris: Gallimard.

Clot, Y. (1999). La fonction psychologique du travail. Paris: PUF.

Clot, Y. (2000). La formation par l'analyse du travail : pour une troisième voie. In B. Maggi (Ed.), Manières de penser, manières d'agir en éducation et en formation (pp. 133-156). Paris: PUF.

Clot, Y. (2004). Action et connaissance en clinique de l'activité. @ctivités, n¹ (1). http:/www.activités.org/ $\mathrm{v} 1 / \mathrm{n} 1$.

Clot, Y. (2005). L'autoconfrontation croisée en analyse du travail : l'apport de la théorie bakhtienne du dialogue. In L. Fillietaz, \& J.P. Bronckart (Eds.), L'analyse des actions et des discours en situation de travail (pp. 37-55). BCILL, Peeters: Louvain-La-Neuve.

Clot Y. et al. (2005). Enjeux du travail et " genres professionnels » dans la recomposition en cours des métiers de la fonction publique. Le cas des professeurs de l'enseignement secondaire et du personnel soignant de gérontologie. Rapport pour le Ministère de la Recherche, ACI « Travail ».

Clot, Y., Faïta, D. Fernandez, G., \& Scheller, L. (2001). Les entretiens en autoconfrontation croisée : une méthode en clinique de l'activité. Éducation Permanente, 146, 17-25.

Clot, Y., Magnier, J., \& Werthe, C. (2000). La validation des acquis, concepts, méthodes, terrain, CPC Documents, 4. Paris: Ministère de l'Education Nationale.

Clot, Y., Prot, B., \& Werthe, C. (Eds.) (2001). Clinique de l'activité et pouvoir d'agir. Education Permanente, $\mathrm{n}^{\circ} 146$.

Diallo, M., \& Clot, Y. (2003). L'exploration de l'expérience dans l'analyse de l'activité. Problèmes et 
méthodes. L'orientation Scolaire et Professionnelle, 32 (2), 203-217.

Lebeaume, J. (Ed.) (1999). Discipline scolaire et prise en charge de l'hétérogénéité. Pratiques enseignantes en technologie au collège. Rapport de recherche en réponse à l'appel d'offre du Comité National de Coordination de la Recherche en Education, G.D.S.T.C.-L.I.R.E.S.T., ENS Cachan.

Léontiev, A. (1984). Activité, conscience, personnalité. Moscou: Editions du Progrès.

Martinand, J.-L. (1986). Connaître et transformer la matière. Berne: Peter Lang.

Plazaola Gige, I. (2004). Prescrire l'agir enseignant? Le cas de l'allemand à l'école primaire genevoise. In J.-P. Bronckart (Ed.), Agir et discours en situation de travail, Cahiers de Recherches des Sciences de l'Education, $\mathrm{n}^{\circ} 103,185-211$.

Prot, B. (2003). Le concept potentiel : une voie de développement des concepts. Le cas de la validation des acquis. Thèse présentée sous la direction de Y. Clot, Paris: CNAM.

Vermersch, P. (1993). Pensée privée et représentation dans l'action. In A. Weil-Fessina, \& P. Rabardel (Eds.), Représentation pour l'action (pp. 209-232). Toulouse: Octarès Éditions.

Vermersch, P. (1994). L'entretien d'explicitation. Paris: ESF.

Vermersch, P. (1999). Pour une psychologie phénoménologique. Psychologique Française, 44 (1), 7-18.

Vermersch, P. (2005). Éléments pour une méthode de « dessin du vécu » en psycho-phénoménologie. Expliciter, 62, 47-57.

Vygotski, L. (1930/1985). La méthode instrumentale en psychologie. In B. Schneuwly, \& J.-P. Bronckart (Eds.), Vygotsky aujourd'hui (pp. 39-47). Lausanne: Delachaux et Niestlé.

Vygotski, L. (1994). Défectologie et déficience mentale, textes publiés par K. Bariniskov et G. Petitpierre. Lausanne: Delachaux et Niestlé.

\section{RÉSUMÉ}

Dans cet article, on présente un dialogue en autoconfrontation croisée entre deux professeurs de technologie en introduisant une différence entre «mémoire privée du travail » et " mémoire collective du métier ». On s'efforce de montrer que la mobilisation du genre professionnel et le recours à la mémoire du métier que cela implique dans les échanges entre pairs et chercheurs favorisent et développent les interprétations à propos des règles de métier, permettant ainsi de soutenir et relancer des débats de métier que les professionnels considéraient comme épuisés.

\section{Mots CLÉ}

Activité, dialogue, expérience, genre, métier, travail.

\section{RESUMEN}

En este artículo, se presenta un diálogo de auto confrontación cruzada entre dos profesores de tecnología, introduciendo una diferencia entre « memoria privada del trabajo » $\mathrm{y}$ «memoria colectiva del oficio ». Se hace hincapié en mostrar que, en los intercambios entre pares e investigadores, la movilización del género profesional y el recurso a la memoria del oficio que esto implica, favorecen y desarrollan las interpretaciones acerca de las reglas del oficio, permitiendo así sostener y relanzar los debates acerca del oficio, que los profesionales creían ya agotados.

Palabras Clave

Actividad, diálogo, experiencia, género, oficio, trabajo 
Article reçu le 25 mai 2005, accepté le 13 Septembre 2006 